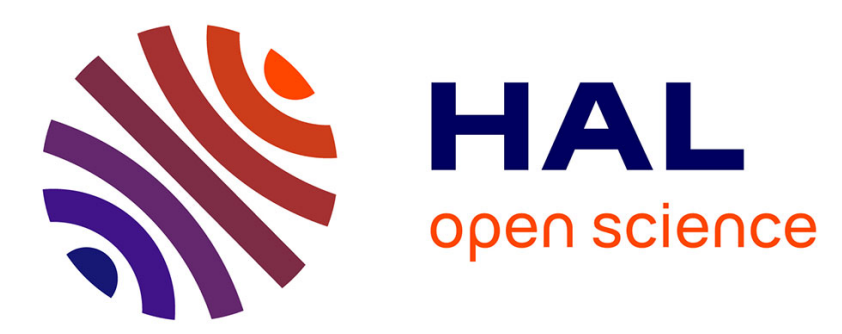

\title{
A Reactive Control Approach for Pipeline Inspection with an AUV
}

Pedro K. Paim, Bruno Jouvencel, Lionel Lapierre

\section{To cite this version:}

Pedro K. Paim, Bruno Jouvencel, Lionel Lapierre. A Reactive Control Approach for Pipeline Inspection with an AUV. OCEANS, Sep 2005, Washington, DC, United States. pp.201-206, 10.1109/OCEANS.2005.1639762 . lirmm-00106473

\section{HAL Id: lirmm-00106473 \\ https://hal-lirmm.ccsd.cnrs.fr/lirmm-00106473}

Submitted on 16 Oct 2006

HAL is a multi-disciplinary open access archive for the deposit and dissemination of scientific research documents, whether they are published or not. The documents may come from teaching and research institutions in France or abroad, or from public or private research centers.
L'archive ouverte pluridisciplinaire HAL, est destinée au dépôt et à la diffusion de documents scientifiques de niveau recherche, publiés ou non, émanant des établissements d'enseignement et de recherche français ou étrangers, des laboratoires publics ou privés. 


\title{
A Reactive Control Approach for Pipeline Inspection with an AUV
}

\author{
Pedro K. Paim*, Bruno Jouvencel ${ }^{\dagger}$ and Lionel Lapierre ${ }^{\dagger}$ \\ *Laboratório de Controle e Visão por Computador \\ Departamento de Engenharia Elétrica (ENE) - Universidade de Brasília, \\ Caixa Postal 04591 - Asa Norte - Brasília - CEP 70910-900 - Brazil \\ Email: pedropaim@terra.com.br \\ ${ }^{\dagger}$ Département Robotique - LIRMM \\ UMR CNRS/Université de Montpellier II, $n^{\circ}$ C55060 \\ 161, rue Ada 34392 - Montpellier - Cedex 5 - France \\ Email: Bruno.Jouvencel@lirmm.fr \\ Email: lapierre@lirmm.fr
}

\begin{abstract}
Recent technological advances and the growing demands for underwater operations suggest that autonomous underwater vehicles will become increasingly present in commercial and research activities, performing tasks such as survey, inspection of sub-sea pipelines and object recovery. This fact calls for the development of guidance and control algorithms for the execution of this kind of mission. This paper proposes a reactive control approach for pipeline following by a torpedolike autonomous underwater vehicle. The method presented here, called the Deformable Virtual Zones (DVZ) method was originally conceived at the LIRMM for obstacle avoidance by mobile robots and AUVs. It consists of the modelling of a virtual zone around the robot, whose configuration depends on the robots states. The intrusion of proximetric information in this zone inflicts a deformation, and the control signal is computed so that the robot will react in order to minimise this deformation. We extend this sensor-based control method by proposing a new DVZ configuration for pipeline following by an autonomous underwater vehicle. Simulation results allow verifying its robustness as the pipeline changes direction, taking into account AUV dynamics. The proposed method is currently being implemented using the TAIPAN II, a small sized torpedolike AUV developed at the LIRMM, fitted with a profiling sonar system. Experimental results will soon be available.
\end{abstract}

\section{INTRODUCTION}

Driven by the growing demands for underseas operations in the fields of telecommunication and oil extraction, underwater robotics is rapidly developing. Underwater robots, or UUVs (unmanned underwater vehicles) have long been present performing commercial operations, military missions or aiding in oceanographic research. They perform tasks such as inspection of undersea equipment, object recovery, maintenance and mine detection among others, at depths where it would be too hazardous or impractical for manned vehicles.

Autonomous underwater vehicles, or $A U V s$, have become a new tendency on a realm traditionally dominated by ROVs (remotely operated vehicles), which are controlled from a distance through an umbilical cable. Recent technological advances suggest that inspection of undersea equipment, pipelines and cables will soon be routinely carried out by AUVs. This fact motivates the development of guidance and control algorithms for this kind of mission.

This paper discusses the application of a reactive control method for AUVs. The method presented here, called the Deformed Virtual Zones (DVZ), was conceived at the LIRMM for obstacle avoidance by mobile robots, but has been investigated in other fields aswell. It consists of the modeling of an imaginary protective zone around the robot which can be deformed by the intrusion of obstacles or objects. The aim is that the robot react in such a way as to minimize this deformation, thus avoiding collision with the obstacle.

What makes this method appealing is that it requires no modeling of the objects, only of the robot's interaction with its environment. Once the virtual zone and its desired behaviour have been described mathematically, the computation of the control law becomes relatively simple and computationally inexpensive.

Here, we investigate the application of the DVZ method for the problem of an AUV following an underwater pipeline for inspection. We propose a three-dimensional DVZ configuration and we present a set of simulation results.

The paper begins by presenting TAIPAN I and II, the two prototype AUVs used in research at the LIRMM, section II. A brief description of the research activities conducted is also given. Subsequently, section III describes the mathematical modeling of TAIPAN's dynamics.

The deformed virtual zones method is introduced in section IV, as we describe its general form for obstacle avoidance. Section V discusses the application of the DVZ for pipeline following.

\section{THE TAIPAN AUV}

\section{A. Description of the prototypes}

The LIRMM relies on two prototype AUVs, TAIPAN I and II, for its research activities. Both are low-cost, torpedo- 
shaped vehicles. They differ from one another in dimension and hardware. Table I shows the dimensions and weight of the two prototypes.

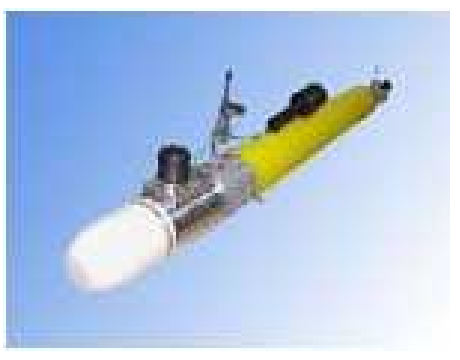

(a) TAIPAN I

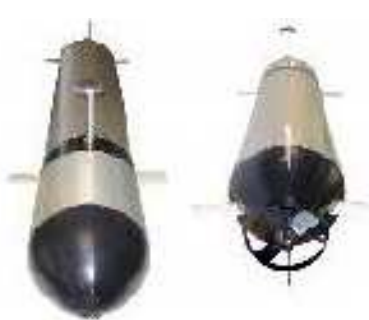

(b) TAIPAN II
Fig. 1. TAIPAN I and II

Both vehicles have a single propeller, a rudder and a stern diving plane. An additional diving plane, located in the front, endows the vehicle with new capabilities and suits it for very shallow water applications. These additional control surfaces provide extra lift, which allows controlling the vehicle down to 2 knots. The specificity of TAIPAN I and II for shallow water operation also lies in its ability to change depth at zero pitch, and to dive from the surface by itself, by appropriate control of the two diving planes.

To compute displacement, the prototypes rely on inertial navigation systems containing inclinometers, magnetometers, yaw rate and pitch rate girometers, pressor sensors and GPS receivers. Power is provided by $48 \mathrm{~V} / 8 \mathrm{Ah}$ and $48 \mathrm{~V} / 16 \mathrm{Ah}$ NiMH battery packs respectively for TAIPAN I and II.

The hardware on TAIPAN I is based on an INMOS T805 transputer board with additional components dedicated to A/D conversions, RS232 serial communication, digital I/O and PWM signal generation. A more detailed description TAIPAN I is found in [6].

TAIPAN II, the more recent version, uses a PENTIUM III processor. It is equipped with a Lock Doppler to aid in displacement estimation, an UHF radio link, a WiFi link and an accoustical modem for communication and an accoustical range meter and GPS receiver for dead-reckoning. It employs three accoustical sensors for obstacle avoidance. The scientific payload can be located in the nose, including a conductivity, temperature and depth sensor and an accoustical doppler current profiler.

\section{B. Research activities in Underwater Robotics}

The underwater robotics group at the LIRMM currently focuses on robust control, high-level task planning and multiple AUV coordination. The application of high-order sliding-mode

TABLE I

DIMENSIONS OF TAIPAN I AND II

\begin{tabular}{c|c|c|c}
- & Length & Diameter & Weight \\
\hline TAIPAN I & $1,66 \mathrm{~m}$ & $0,15 \mathrm{~m}$ & $25 \mathrm{~kg}$ \\
TAIPAN II & $1,80 \mathrm{~m}$ & $0,20 \mathrm{~m}$ & $60 \mathrm{~kg}$
\end{tabular}

control, using techniques such as twisting and super-twisting, has been described in [4][5].

The integration of these lines of research will take place when a complex task can be efficiently accomplished by a fleet of AUVs. One envisioned application would be the inspection of underwater pipelines by a a pair of AUVs working in cooperation. The vehicles would be released from a boat, or mother-ship. The first AUV would have the role of leader, following the pipeline at short distance. The collected information would be transmitted to the second AUV, the follower, closer to the surface. At certain intervals, the follower would emerge and retrasmit its data to the mother-ship, while also updating its GPS information.

The use of multiple AUVs instead of a single one would represent lower energy consumption and increased efficiency. The follower AUV, closer to the surface, would eliminate the need for the main vehicle to emerge to retransmit data and update the GPS information, which would require that it move all the way to the surface and then back. The elimination of this need would mean considerable energy savings.

Furthermore, the operation of the profiling sonar drains a lot from the battery. In a situation where more than one vehicle is used, they could exchange the role of leader and follower, making better use of the energy available. This could increase mission autonomy.

\section{MATHEMATICAL Modeling}

According to the common practice in underwater robotics, the six-degree-of-freedom dynamics of TAIPAN are represented using a global coordinate frame and a local one[2](c.f.,Figure 2). This is the representation suggested by the SNAME (Society of Naval Architects and Marine Engineers).

We place the local coordinate frame on the vehicle's center of mass, oriented so that its axes will coincide with the vehicle's main axes of inertia. By doing so, we take advantage of the vehicle's symmetry, leading to a simpler model. This system has velocity components given by the linear velocity vector $\mathbf{v}_{1}=[u, v, w]^{T}$ and the angular velocity vector $\mathbf{v}_{2}=$ $[p, q, r]^{T}$. The general velocity vector is represented as:

$$
\mathbf{v}=\left[\mathbf{v}_{1}^{T}, \mathbf{v}_{2}^{T}\right]=[u, v, w, p, q, r]^{T} .
$$

The position and orientation vectors $\eta_{1}=[x, y, z]^{T}$ and $\eta_{2}=[\phi, \theta, \psi]^{T}$ are expressed with respect to the global coordinate frame. In its concise form:

$$
\eta=\left[\eta_{1}^{T}, \eta_{2}^{T}\right]=[x, y, z, \phi, \theta, \psi]^{T},
$$

where $\phi$ is the roll angle, $\theta$ is the pitch angle and $\psi$ is the yaw.

\section{A. Kinematics}

The velocities in the local frame are related to those in the global frame by the expression:

$$
\left[\begin{array}{c}
\dot{\eta_{1}} \\
\dot{\eta_{2}}
\end{array}\right]=\left[\begin{array}{cc}
J_{1}\left(\eta_{2}\right) & 0 \\
0 & J_{2}\left(\eta_{2}\right)
\end{array}\right]\left[\begin{array}{l}
v_{1} \\
v_{2}
\end{array}\right],
$$



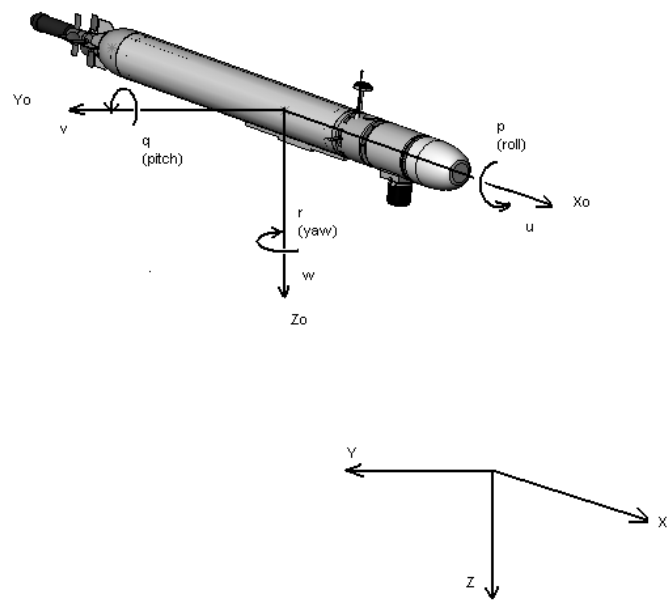

Fig. 2. Sistemas de coordenadas do TAIPAN

where $J_{1}\left(\eta_{2}\right)$ is the transformation matrix which yields the linear velocity components of $v_{1}$ in the global frame, and $J_{2}\left(\eta_{2}\right)$ maps the angular velocities $v_{2}$ into the global frame.

\section{B. Dynamics}

The dynamics of underwater vehicles are modelled based on the priciples of rigid-body motion. They're determined by the vehicle's inertia, coriolis and centrifugal forces as well as by hydrodynamic forces, due to the relative motion between the vehicle and its surrounding fluid. In order to take into account the inertia of the fluid, i.e. the exchange of kinetic energy between the vehicle and the fluid, we use the concept of added mass.

The six non-linear Newton-Euler equations of motion can be expressed in a compact fashion as [2]:

$$
\mathbf{M} \dot{\mathbf{v}}+\mathbf{C}(\mathbf{v}) \mathbf{v}+\mathbf{D}(\mathbf{v}) \mathbf{v}+\mathbf{g}(\eta)=\tau
$$

where $\mathbf{M}=\mathbf{M}_{R B}+\mathbf{M}_{A}$ is the inertia matrix with added mass, $\mathbf{C}=\mathbf{C}_{R B}+\mathbf{C}_{A}$ is the matrix of coriolis and centripetal terms , $\mathbf{D}$ is the damping matrix, $\mathbf{g}$ is the vector of gravitational forces and moments, and $\tau$ is the vector of actuator forces and moments, for which we use linearized models.

\section{The Deformed VirTual Zones}

\section{A. A general description}

The Deformed Virtual Zones (DVZ) method is a sensorbased control method, originally developed at the LIRMM for obstacle avoidance by mobile robots [7], but whose applications have been extended to other fields, such as aerial robotics [8]. Other applications of reactive control are described in [1], [9] and [3].

This method consists of the modeling of an imaginary protective region, called the DVZ, around the robot, whose configuration depends on the robot's states. Surrounding objects are detected by proximity sensors. When an object

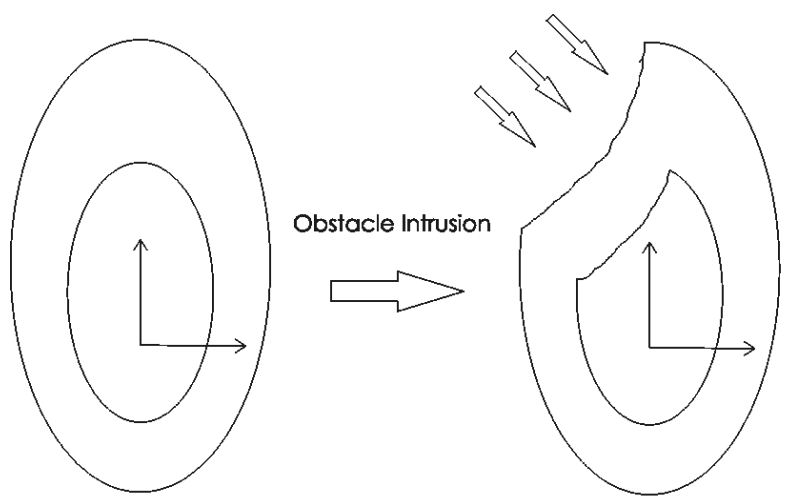

Fig. 3. DVZ Deformation

penetrates this protective zone, it inflicts a deformation and the control signal is then calculated so that the robot react in such a way that minimizes the deformation on the DVZ.

The DVZ's greatest advantage lies in the fact that it requires no modeling of the objects, only modeling of the robot's interaction with its environment. Once the virtual zone and its desired behaviour have been described mathematically, the computation of the control law becomes relatively simple and computationally inexpensive.

For obstacle avoidande, the DVZ is modelled as an ellipse, in the two-dimensional case, or an ellipsoid, if the vehicle moves in three-dimensional space. The parameters of this ellipse depend on the robot's states. It can therefore become larger as the robot moves faster, meaning the robot would detect and react to objects which are further away. Figure 3 shows a situation where a robot approaches an obstacle, which deforms the DVZ. This deformation can be minimized by a combination of two actions: a reduction in its forward velocity and a change in orientation. Figure 4 shows the robot's attempt to minimize the deformation by changing its orientation.

The DVZ is expressed by a sum of two terms which describe the interaction of the robot and its environment:

$$
\Xi=\Xi_{h}+\Delta \text {. }
$$

where $\Xi_{h}$ represents the undeformed protective zone and $\Delta$ represents a deformation due to proximity information.

The geometry of the DVZ is a function of the vector $\pi$, which characterize the robot's controllable states:

$$
\Xi_{h}=\rho_{\Xi}(\pi)
$$

The deformation depends on the intrusion of proximity information and on the configuration of the non-deformed protective zone:

$$
\Delta=\alpha\left(\Xi_{h}, I\right) .
$$

The derivative of this deformation is:

$$
\dot{\Delta}=\left(\frac{\partial \alpha}{\partial \Xi_{h}}\left(\Xi_{h}, I\right) \times \rho_{\Xi}^{T}(\pi)\right) \dot{\pi}+\frac{\partial \alpha}{\partial I}\left(\Xi_{h}, I\right) \dot{I} .
$$




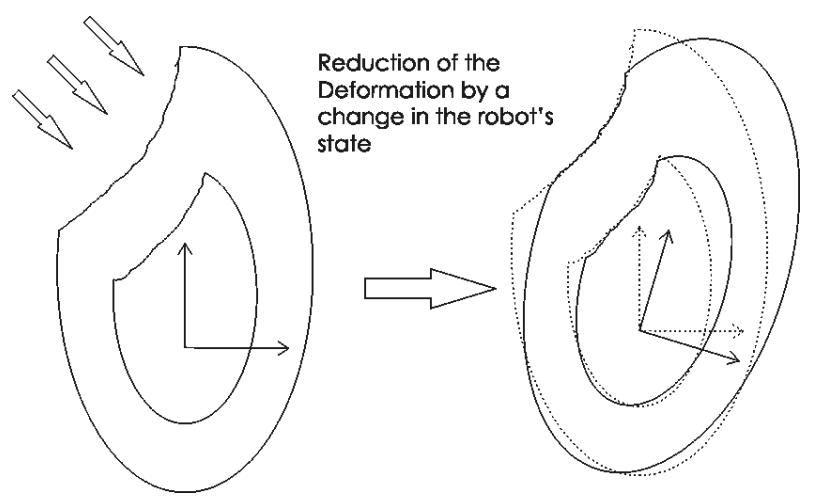

Fig. 4. Reduction of the deformation by changing orientation

It can be observed that the evolution of the virtual zone's deformation is determined by two factors. The first one is caused by the vector $\dot{\pi}$, which represents the robot's controllable states. The second one, $\dot{I}$, is caused by the environment itself. The objective of the DVZ method is to calculate the control vector $\dot{\pi}$ so as to minimize the deformation.

The complete evolution of the DVZ can also be expressed as:

$$
\dot{\Delta}=A(\pi, I) \dot{\pi}+B(\pi, I) \dot{I}
$$

The first step in computing the control law is to determine a desired evolution for the deformation. One appropriate solution is a weighted sum of the deformation itself and its derivative with respect to time:

$$
\nabla=-M \Delta-N \dot{\Delta}
$$

Keeping in mind that $A(\pi, I)$ is the function which relates the robot's states with the DVZ's deformation, the control signal which will minimize this deformation is computed as

$$
\phi_{\text {best }}=A^{+} \cdot \nabla
$$

where $A^{+}=\left(A^{T} A\right)^{-1} A^{T}$ is the Moore-Penrose pseudoinverse of $A(\pi, I)$. This will yield a control vector which leads to the desired evolution of the deformation $\nabla$, in the least-squares sense.

\section{B. Simulation}

The simulation shown in figure 5 employs the principles decribed above. It represents a single AUV moving in an unknown environment in an attempt to reach a final destination. Starting point, mission waypoint and final destination are determined by the user, as are the number of proximity sensors used and the number of obstacles, which are represented by the larger circles. The figure shows the satisfactory performance of the DVZ, obtained at low computational cost.

\section{THE DVZ IN PIPELINE INSPECTION}

The extension of the Deformed Virtual Zones method to the problem of an AUV following a pipeline begins with the conception of a new DVZ configuration such that the continuous effort to minimize its deformation will cause the

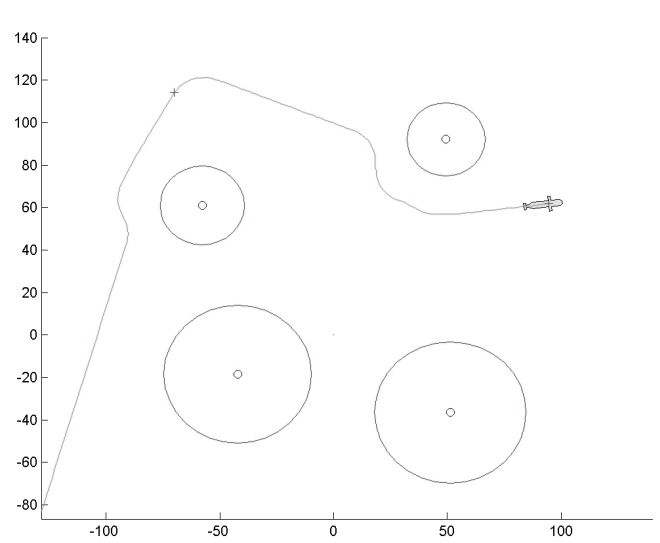

Fig. 5. Obstacle avoidance with an AUV

vehicle to follow the pipeline. So, the chosen DVZ configuration is a triangular prism with a cylindrical cavity below the AUV. In case the robot leaves its trajectory, the pipe becomes misaligned with the cavity, deforming the virtual zone.

We assume that the robot is fitted with a profiling sonar attached to its head, giving readings of the profile of the pipe and of the seafloor. Since the profiling sonar only gives readings on a plane perpendicular to its length, the control law must be computed based on the projection of the DVZ onto the sensor plane.

Figure 6 shows the three-dimensional configuration of the DVZ for pipeline following and its projection on the sensor plane. $\{R\}$ denotes a robot-fixed coordinate frame and $\{\mathrm{DVZ}\}$ denotes a DVZ-fixed coordinate frame. It can be seen how the cavity becomes elliptical and misaligned as the DVZ-fixed frame is rotated. We need to find the analytical expression of the DVZ and differentiate it with respect to all the vehicle's controllable states. The result of this differentiation must reflect the change of radius of the DVZ in the direction of each individual sensor caused by the misalignment between the two coordinate frames.

\section{A. The Discrete DVZ and the Computation of the Control Law}

We consider that the profiling sonar yields $n$ proximity readings along equally spaced angles $\left(\alpha_{1}, \alpha_{2}, \ldots, \alpha_{n}\right)$. The Jacobian matrix which relates the variation of the deformation on the DVZ and the variation of each of the vehicle's controllable states is:

$$
J=\left[\begin{array}{ccccc}
\frac{\partial Y\left(\alpha_{1}\right)}{\partial u} & \frac{\partial Y\left(\alpha_{2}\right)}{\partial u} & \ldots & \frac{\partial Y\left(\alpha_{n-1}\right)}{\partial u} & \frac{\partial Y\left(\alpha_{n}\right)}{\partial u} \\
\frac{\partial Y\left(\alpha_{1}\right)}{\partial w} & \frac{\partial Y\left(\alpha_{2}\right)}{\partial w} & \cdots & \frac{\partial Y\left(\alpha_{n-1}\right)}{\partial w} & \frac{\partial Y\left(\alpha_{n}\right)}{\partial w} \\
\frac{\partial Y\left(\alpha_{1}\right)}{\partial \psi} & \frac{\partial Y\left(\alpha_{2}\right)}{\partial \psi} & \cdots & \frac{\partial Y\left(\alpha_{n-1}\right)}{\partial \psi} & \frac{\partial Y\left(\alpha_{n}\right)}{\partial \psi} \\
\frac{\partial Y\left(\alpha_{1}\right)}{\partial \theta} & \frac{\partial Y\left(\alpha_{2}\right)}{\partial \theta} & \ldots & \frac{\partial Y\left(\alpha_{n-1}\right)}{\partial \theta} & \frac{\partial Y\left(\alpha_{n}\right)}{\partial \theta} \\
\frac{\partial Y\left(\alpha_{1}\right)}{\partial \phi} & \frac{\partial Y\left(\alpha_{2}\right)}{\partial \phi} & \cdots & \frac{\partial Y\left(\alpha_{n-1}\right)}{\partial \phi} & \frac{\partial Y\left(\alpha_{n}\right)}{\partial \phi}
\end{array}\right] ;
$$

where $Y(\alpha)$ is the DVZ's deformation along the direction $\alpha$, i.e. the difference between the non-deformed DVZ and the sensor reading in that direction. 

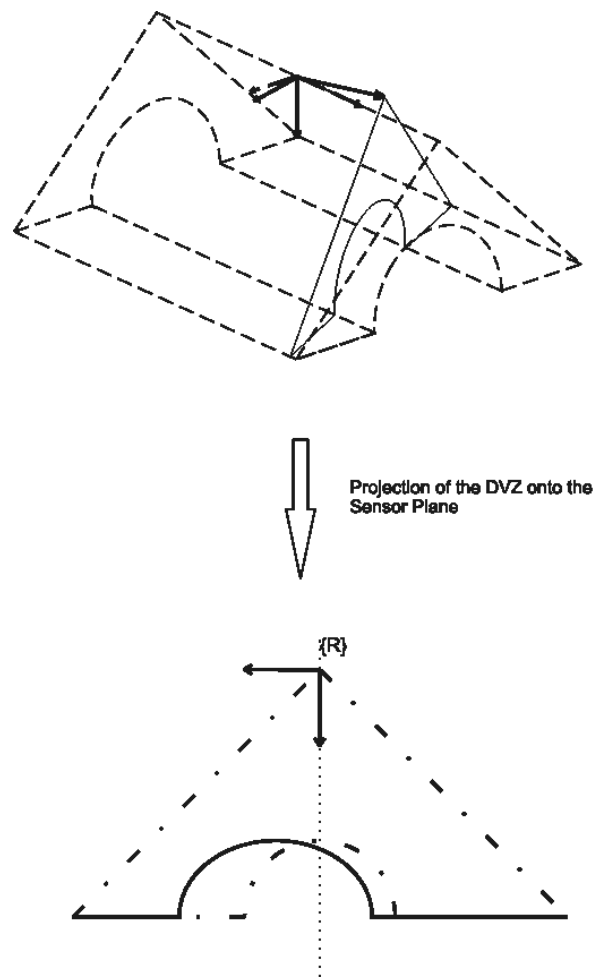

Fig. 6. Projection of the DVZ onto the sensor plane

Nonetheless, for the present application, it is desired that the robot maintain constant velocity and null pitch and roll angles. Thus these states are not taken into account and the Jacobian matrix is reduced to:

$$
J=\left[\begin{array}{lllll}
\frac{\partial Y\left(\alpha_{1}\right)}{\partial w} & \frac{\partial Y\left(\alpha_{2}\right)}{\partial w} & \cdots & \frac{\partial Y\left(\alpha_{n-1}\right)}{\partial w} & \frac{\partial Y\left(\alpha_{n}\right)}{\partial w} \\
\frac{\partial Y\left(\alpha_{1}\right)}{\partial \psi} & \frac{\partial Y\left(\alpha_{2}\right)}{\partial \psi} & \cdots & \frac{\partial Y\left(\alpha_{n-1}\right)}{\partial \psi} & \frac{\partial Y\left(\alpha_{n}\right)}{\partial \psi}
\end{array}\right]
$$

The control law is calculated as the product of the Jacobian matrix's Moore-Penrose pseudo-inverse and the desired evolution of the DVZ:

$$
\begin{aligned}
\nabla & =-M \Delta-N \dot{\Delta}, \\
\phi_{\text {best }} & =J^{+} \cdot \nabla .
\end{aligned}
$$

Figure 8 presents a set of simulation results, showing yaw control signal, the linear tracking error and the angular tracking error. With a sampling period of 2 seconds, the AUV was capable of following the pipeline even with sudden changes in orientation. When the pipeline changed $45^{\circ}$ in direction, the tracking error did not exceed four meters. Satisfactory performance was obtained with sampling periods up to 8 seconds, after which the system becomes overly unstable. A sonar model to account for measurment uncertainty is yet to be implemented in the simulation.

\section{CONClusion}

In this article, we have provided a brief overview of the research activities conducted at the LIRMM in the field of

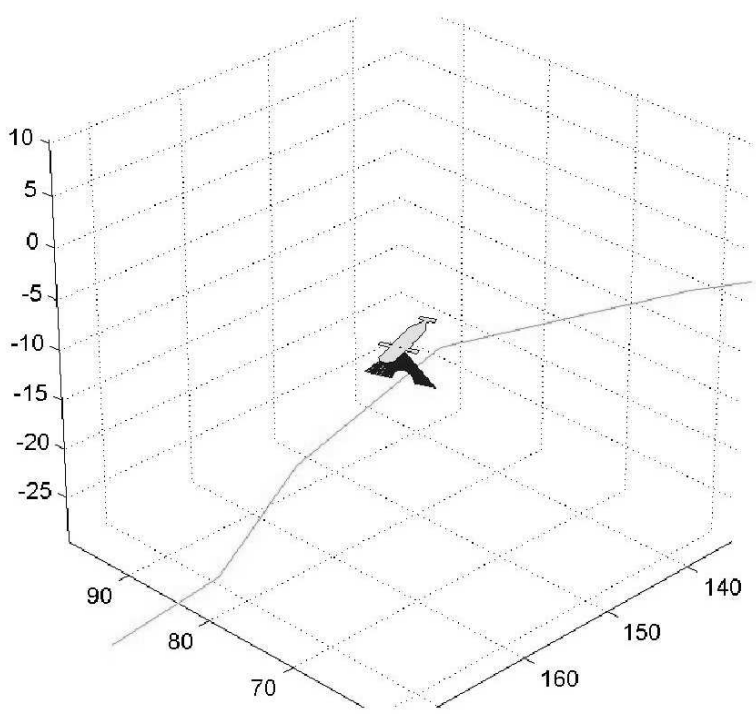

Fig. 7. Simulation of Pipeline Following
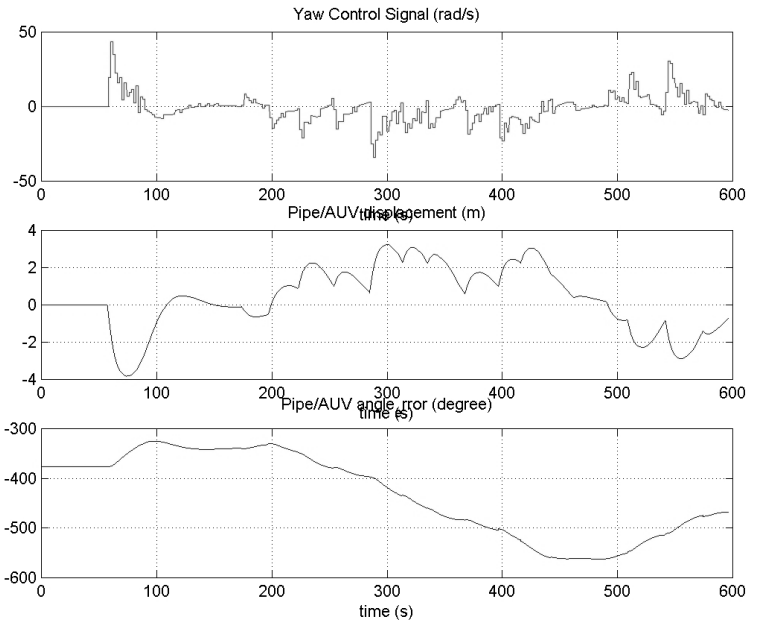

Fig. 8. Simulation Results - Control Signal, Linear Tracking Error, Angular Tracking Error

underwater robotics and we have described the prototype AUVs used at this laboratory.

We have presented the the Deformed Virtual Zones method for obstacle avoidance and we have extended it to the problem of an AUV following an underwater pipeline for inspection. Simulation yielded satisfatory results with various sampling periods, even as the pipeline abruptly changes direction.

The proposed method is currently being implemented on the TAIPAN II AUV fitted with a profiling sonar. First experimental results will be available soon. We are currently investigating the application of sliding-mode control along with the DVZ method, looking to resolve a few limitations of the control law presented herein. 


\section{ACKNOWLEDGMENT}

Thanks to Etienne Dombre, director of the Département Robotique, and Bruno Jouvencel for welcoming author Pedro K. Paim during his 6-month visit to the LIRMM. Author Pedro K. Paim would also like to thank Lionel Lapierre and the remainder of the underwater robotics team for their friendship and cooperation.

\section{REFERENCES}

[1] V. Creuze. Navigation referencée terrain pour véhicule autonome sousmarin. PhD thesis, Université de Montpellier II, November 2002.

[2] T. I. Fossen. Guidance and Control of Ocean Vehicles. John Wiley \& Sons Ltd., 1994.

[3] P. Lépinay, R. Zapata, and B. Jouvencel. Sensor-based control of reactive behaviours of walking machines. In International Conference IECON'93, Maui, Hawaii, USA, December 1993.

[4] T. Salgado-Jimenez. Contribution à la commande d'un robot sous-marin de type torpille. PhD thesis, Univestité de Montpellier II, December 2004.

[5] T. Salgado-Jimenez, J-M. Spiewak, P. Fraisse, and B. Jouvencel. A robust control algorithm for auv: based on a high order sliding mode. In Proceedings IEEE OCEANS, Kobe, Japan, 2004.

[6] J. Vaganay, B. Jouvencel, and P. Lépinay. Taipan, an auv for very shallow water applications. In World Automation Congress, San Diego, 1998.

[7] R. Zapata. Quelques aspects topologiques de la planification de mouvements et des actions réflexes en robotique mobile. $\mathrm{PhD}$ thesis, Université de Montpellier II, July 1991.

[8] R. Zapata and P. Lépinay. Collision avoidance of a 3d simulated flying robot. In International Symposium in Robotics and Automation, 1998.

[9] R. Zapata, P. Lépinay, and P. Thompson. Reactive behaviours of fast mobile robots. Journal of Robotic Systems, January 1994. 